\title{
Identification of SMCHD1 domains for nuclear localization, homo-dimerization, and protein cleavage
}

\author{
Yosuke Hiramuki and Stephen J. Tapscott ${ }^{*}$ (i)
}

\begin{abstract}
Background: SMCHD1 is a disease modifier and a causative gene for facioscapulohumeral muscular dystrophy (FSHD) type 1 and type 2, respectively. A large variety of different mutations in SMCHD1 have been identified as causing FSHD2. In many cases, it is unclear how these mutations disrupt the normal function of SMCHD1.

Methods: We made and analyzed lenti-viral vectors that express Flag-tagged full-length or different mutant SMCHD1 proteins to better understand the functional domains of SMCHD1 in muscle cells.

Results: We identified regions necessary for nuclear localization, dimerization, and cleavage sites. Moreover, we confirmed that some mutants increased DUX4 expression in FSHD1 myoblasts.

Conclusions: These findings provide an additional basis for understanding the molecular consequences of SMCHD1 mutations.

Keywords: Facioscapulohumeral muscular dystrophy, SMCHD1, Nuclear localization, Homo-dimerization, Protein cleavage, DUX4
\end{abstract}

\section{Background}

Facioscapulohumeral muscular dystrophy (FSHD) is characterized by weakness initially of the facial, scapular, and upper arm muscles, but progresses to involve most of the skeletal muscles of the body. DUX4 is normally not expressed in the skeletal muscle, whereas it is mis-expressed in the FSHD skeletal muscle [1]. DUX4 is a retrogene encoding a double-homeobox transcription factor and is present in each copy of the D4Z4 macrosatellite repeat, a 3.3 kilobase unit in multicopy arrays in the subtelomeric regions of chromosomes 4 and 10. The most common form of FSHD is caused by a shortened D4Z4 array with ten or fewer units on a permissive haplotype of chromosome 4 (FSHD1) [2, 3]. A phenotypically identical form of FSHD2 is caused by mutations in SMCHD1, a member of the condensin/cohesin family of chromatin factors required for silencing some repetitive regions [4]. FSHD2 also requires the presence of a permissive haplotype of chromosome 4, which is characterized by specific single-nucleotide polymorphisms that create a polyadenylation signal for the DUX4 mRNA [3]. In addition, SMCHD1 is also a disease modifier of FSHD1 [5].

The human SMCHD1 gene consists of 48 exons, and the protein has an ATPase domain in the amino-terminus and a hinge domain in the carboxy-terminus [6]. The ATPase domain hydrolyzes ATP and the hinge domain mediates SMCHD1 dimerization [7-11]. SMCHD1 is a chromatin binding protein that has a role in the epigenetic silencing of the D4Z4 region, the X chromosome, and other regions of DNA repeats in the genome $[4,10,12-$ 16]. Although multiple different FSHD2-causing mutations in SMCHD1 have been reported, limited knowledge of the functional regions of the SMCHD1 protein restrict our understanding of the consequences of each mutation. Here, to better understand the function of SMCHD1 as a chromatin binding protein, we focused on the identification of the region(s) necessary for nuclear localization and homo-dimerization.

* Correspondence: stapscot@fredhutch.org

Human Biology Division, Fred Hutchinson Cancer Research Center, Seattle, WA 98109, USA

(c) The Author(s). 2018 Open Access This article is distributed under the terms of the Creative Commons Attribution 4.0 International License (http://creativecommons.org/licenses/by/4.0/), which permits unrestricted use, distribution, and 


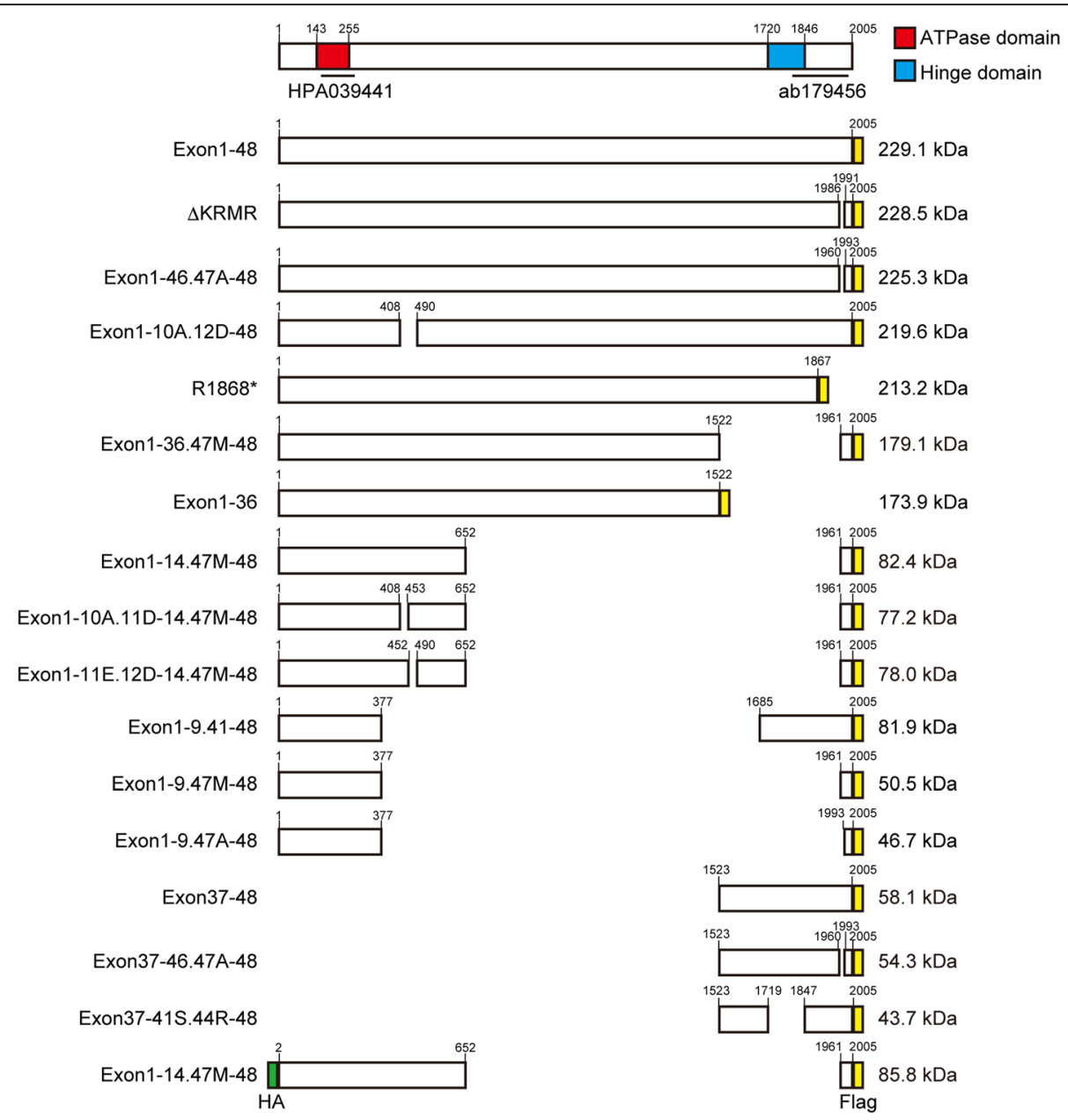

Fig. 1 Schematic of full-length and different mutant SMCHD1 constructs. The gene and protein SMCHD1 consists of 48 exons and 2005 amino acids, respectively, and the ATPase domain in the amino-terminus and the hinge domain in the carboxy-terminus are indicated in red and blue, respectively. A bar indicates recognition regions for anti-SMCHD1 antibody (HPA039441 and ab179456). Predicted molecular weight is shown in the right side. $3 \times \mathrm{HA}$ tag (green) and $3 \times$ Flag tag (yellow)

\section{Methods}

\section{Cell culture}

Control (MB135) and FSHD1 (MB073) myoblasts were cultured in growth medium (F-10 including 10\% fetal bovine serum, $10 \mathrm{ng} / \mathrm{ml}$ human recombinant FGF, $1 \mu \mathrm{M}$ dexamethasone, $100 \mathrm{U} / \mathrm{ml}$ penicillin, and $100 \mu \mathrm{g} / \mathrm{ml}$ streptomycin) or differentiation medium (DMEM including 1\% horse serum, $10 \mu \mathrm{g} / \mathrm{ml}$ insulin, $10 \mu \mathrm{g} / \mathrm{ml}$ transferrin, $100 \mathrm{U} /$ $\mathrm{ml}$ penicillin, and $100 \mu \mathrm{g} / \mathrm{ml}$ streptomycin). These cells were transduced with the lentivirus of interest using $8 \mu \mathrm{g} / \mathrm{ml}$ polybrene. For RT-qPCR, these cells were selected using $2 \mu \mathrm{g} / \mathrm{ml}$ puromycin. For making lentivirus, 293T cells cultured in DMEM including 10\% fetal bovine serum, $100 \mathrm{U} / \mathrm{ml}$ penicillin, and $100 \mu \mathrm{g} / \mathrm{ml}$ streptomycin were transfected with a lenti-viral construct vector, psPAX2 (Addgene plasmid no. 12260), and pMD2.G (Addgene plasmid no. 12259) using lipofectamine 3000 following manufacture protocol. For human SMCHD1 cDNA lenti-viral constructs, cDNA for full length or different mutant SMCHD1 purified by PCR or digested by restriction enzymes was inserted into the GFP site of pLenti CMV GFP Puro (Addgene plasmid no. 17448) [17]. Lenti-viral construct that removed GFP from pLenti CMV GFP Puro was used as "No insert" for RT-qPCR. Sequences of the primers are listed in Additional file 1.

\section{Western blot}

For preparation of sample, cells were lysed with lysis buffer $(50 \mathrm{mM}$ Tris- $\mathrm{HCl} \mathrm{pH} 6.8,150 \mathrm{mM} \mathrm{NaCl}$, $0.2 \%$ Triton $\mathrm{X}-100)$ including protease inhibitors (Roche). Lysates were on ice for $15 \mathrm{~min}$ and spun down at $12000 \mathrm{rpm}$ at $4{ }^{\circ} \mathrm{C}$ for $15 \mathrm{~min}$. The supernatant was transferred to a new tube, added with $2 \times$ Laemmli sample buffer, and boiled for $5 \mathrm{~min}$. For immunoprecipitation (IP), lysates were pre-cleaned with protein $\mathrm{A}$ and protein $\mathrm{G}$ mixture (Millipore) at $4{ }^{\circ} \mathrm{C}$ for $1 \mathrm{~h}$, immunoprecipitated with anti-Flag antibody (Sigma 


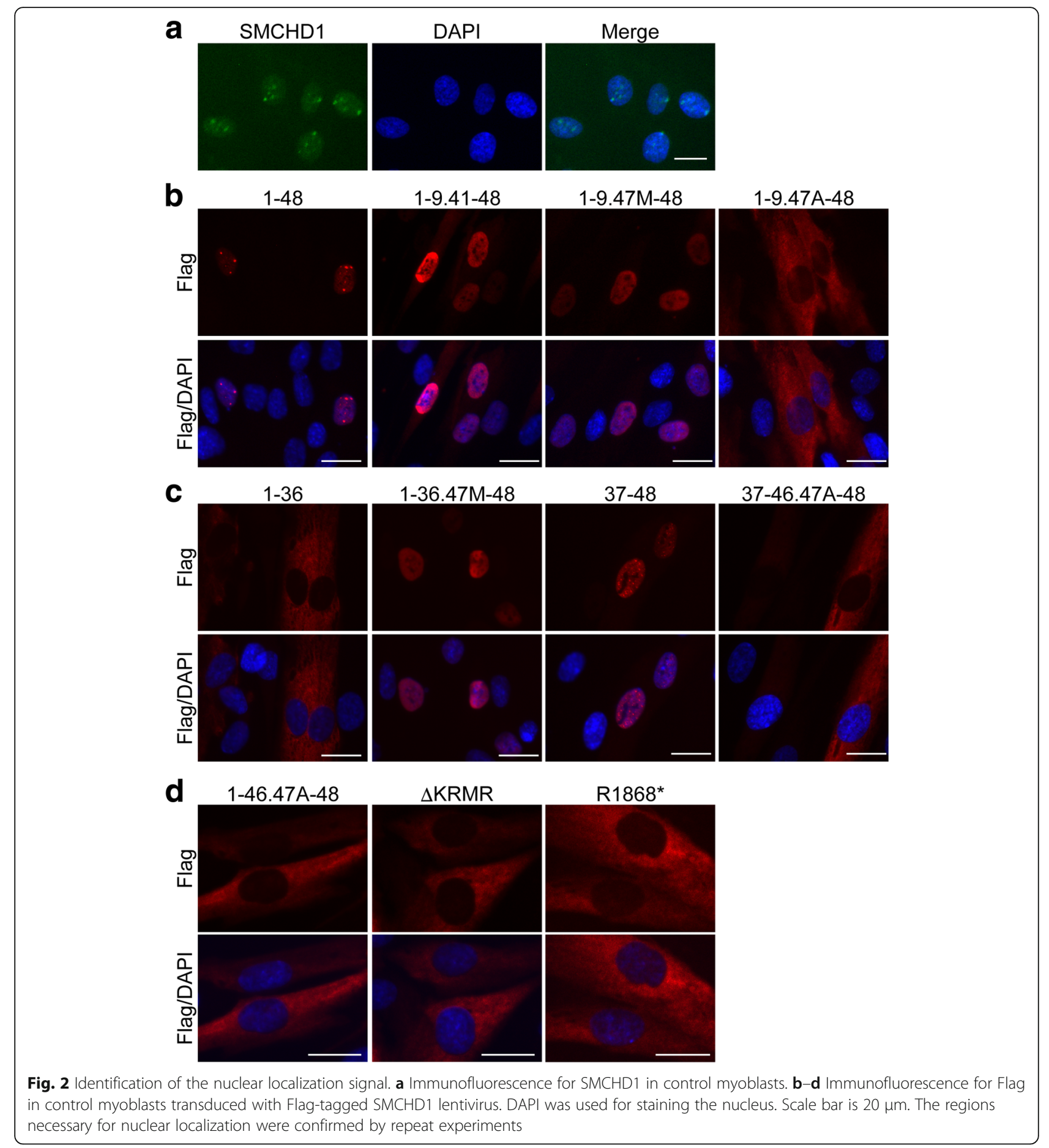

F1804, 1:300) and protein A and protein G mixture at $4{ }^{\circ} \mathrm{C}$ overnight, washed with lysis buffer five times, added with $2 \times$ Laemmli sample buffer, and boiled for $5 \mathrm{~min}$. The sample was resolved on 4-12\% Bis-Tris gel (Invitrogen) and transferred to PVDF membrane (Novex), and then, the membrane was exposed to blocking buffer (5\% dry milk) for $1 \mathrm{~h}$ followed by primary antibody (SMCHD1 (Sigma, HPA039441, 1:3500 or Abcam, ab179456, 1:3500), Flag (Sigma, F1804, 1:3500), HA (Abcam, ab18181, 1:3500), and $\alpha$-Tubulin (Sigma, T9026, 1:14000)) at $4{ }^{\circ} \mathrm{C}$ overnight, and secondary antibody (Jackson ImmunoResearch) at room temperature for $1 \mathrm{~h}$. Signals were detected using ECL Western Blotting Substrate (Thermo Scientific) or West Femto Maximum Sensitivity Substrate (Thermo Scientific) in X-Ray Film Processors (AFP Imaging). 

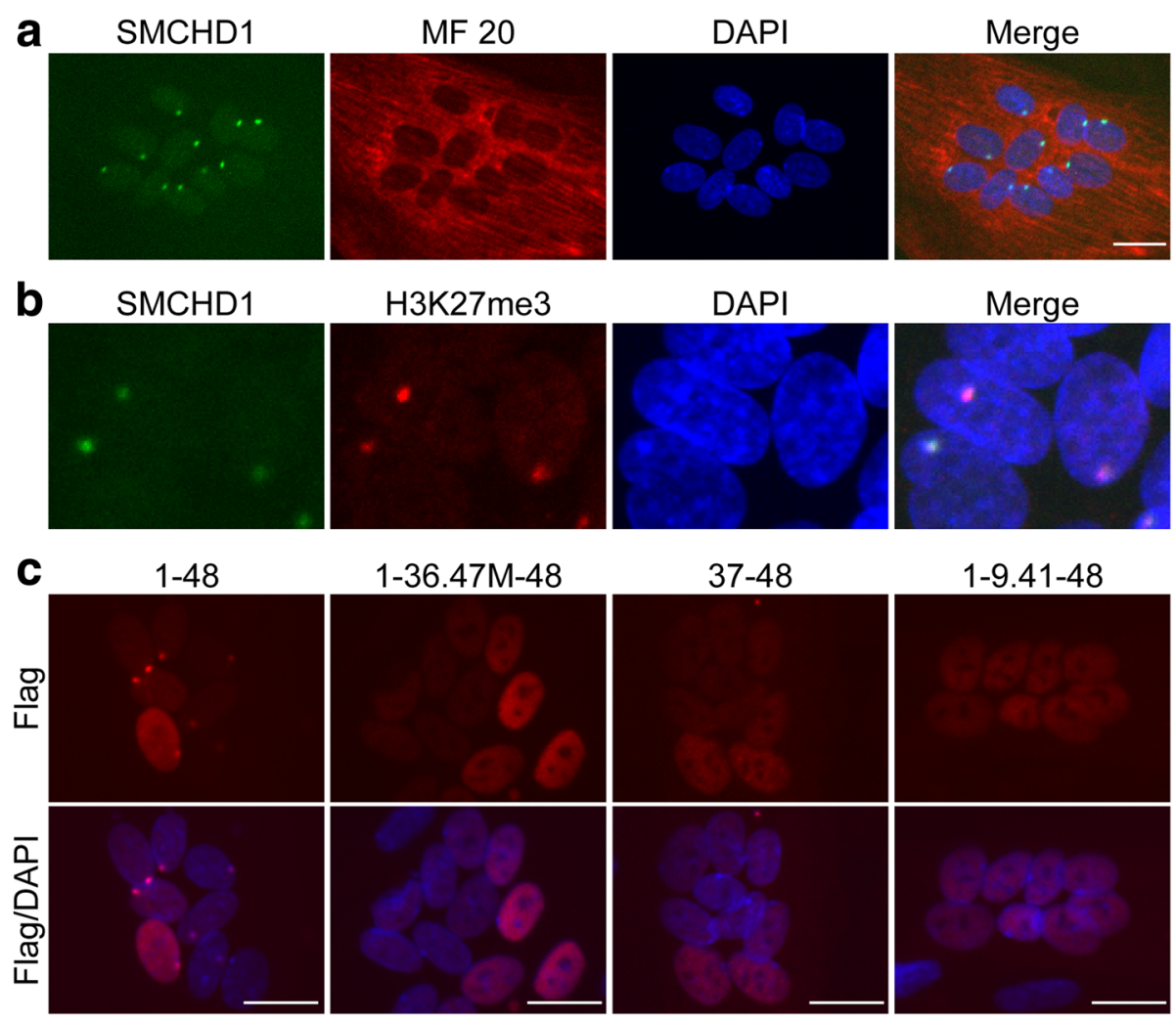

Fig. 3 Single spot of SMCHD1 in female myotube. a Immunofluorescence for SMCHD1 and myosin heavy chain (MF20 antibody) in control female myotubes. b Immunofluorescence for SMCHD1 and H3K27me3 in control female myotubes. c Immunofluorescence for Flag in control female myotubes transduced with Flag-tagged SMCHD1 lentivirus. DAPI was used for staining the nucleus. Scale bar is 20 um. Single spot of full length was confirmed by repeat experiments

\section{Immunofluorescence}

Cells were fixed with 4\% PFA for $12 \mathrm{~min}$, permeabilized with $0.5 \%$ Triton X-100 for 13 min, blocked with blocking buffer $(0.1 \%$ goat serum, $0.1 \%$ BSA, $0.02 \%$ Tween 20$)$, and incubated with primary antibodies (Flag (Sigma, F1804, 1:500), SMCHD1 (Sigma, HPA039441, 1:500), MF20 (DSHB, MF 20 was deposited to the DSHB by Fischman, D.A., 1:500), and H3K27me3 (active motif, 61017, 1:500)) at $4{ }^{\circ} \mathrm{C}$ overnight and incubated with secondary antibodies (Jackson ImmunoResearch) at room temperature for $1 \mathrm{~h}$. DAPI (Sigma) was used for staining nucleic acid. Fluorescence was obtained with a Zeiss Axiophot (AxioCam MRm camera and Axiovision 4.6 software).

\section{RT-qPCR}

Total RNA was isolated using NucleoSpin RNA (Macherey-Nagel). After DNase I treatment, cDNA synthesis with oligo dT primers was performed using SuperScript III Reverse Transcriptase (Invitrogen). After RNase H treatment, RT-qPCR was performed using iTaq Universal SYBR Green Supermix (Bio-Rad) on QuantStudio 7 Flex (Applied Biosystems). Sequences of the primers are listed in Additional file 2 .

\section{Statistical analysis}

Statistical analysis was carried out using Dunnett' s test. A value of $P<0.01$ was considered statistically significant.

\section{Results}

Identification of the SMCHD1 nuclear localization signal In order to characterize SMCHD1 proteins in muscle cells, we made lenti-viral vectors that express full length or different mutant SMCHD1 protein (Fig. 1). SMCHD1 localized to the nucleus in control (MB135) myoblasts under growth medium (Fig. 2a). To identify the nuclear localization signal (NLS) in SMCHD1, we used immunofluorescence to determine the localization of Flag-tagged full-length and mutant SMCHD1 proteins in control myoblasts. The mutant proteins from constructs Exon1-48-Flag, Exon1-9.41-48-Flag, and Exon1-9.47M-48-Flag were mostly localized to the nucleus, whereas proteins from construct Exon1-9.47A-48-Flag were localized to the cytoplasm (Fig. 2b). Therefore, the 32 amino acids (aa) between residues 1961 and 1992 (MTPIRKCNDSLRHSPKVETTDCPVPPKRMRRE) functioned as an NLS. This NLS was sufficient for SMCHD1 to localize to the nucleus because Exon1-36-Flag was expressed in the cytoplasm and Exon1-36.47M-48-Flag, which has NLS sequence, was 

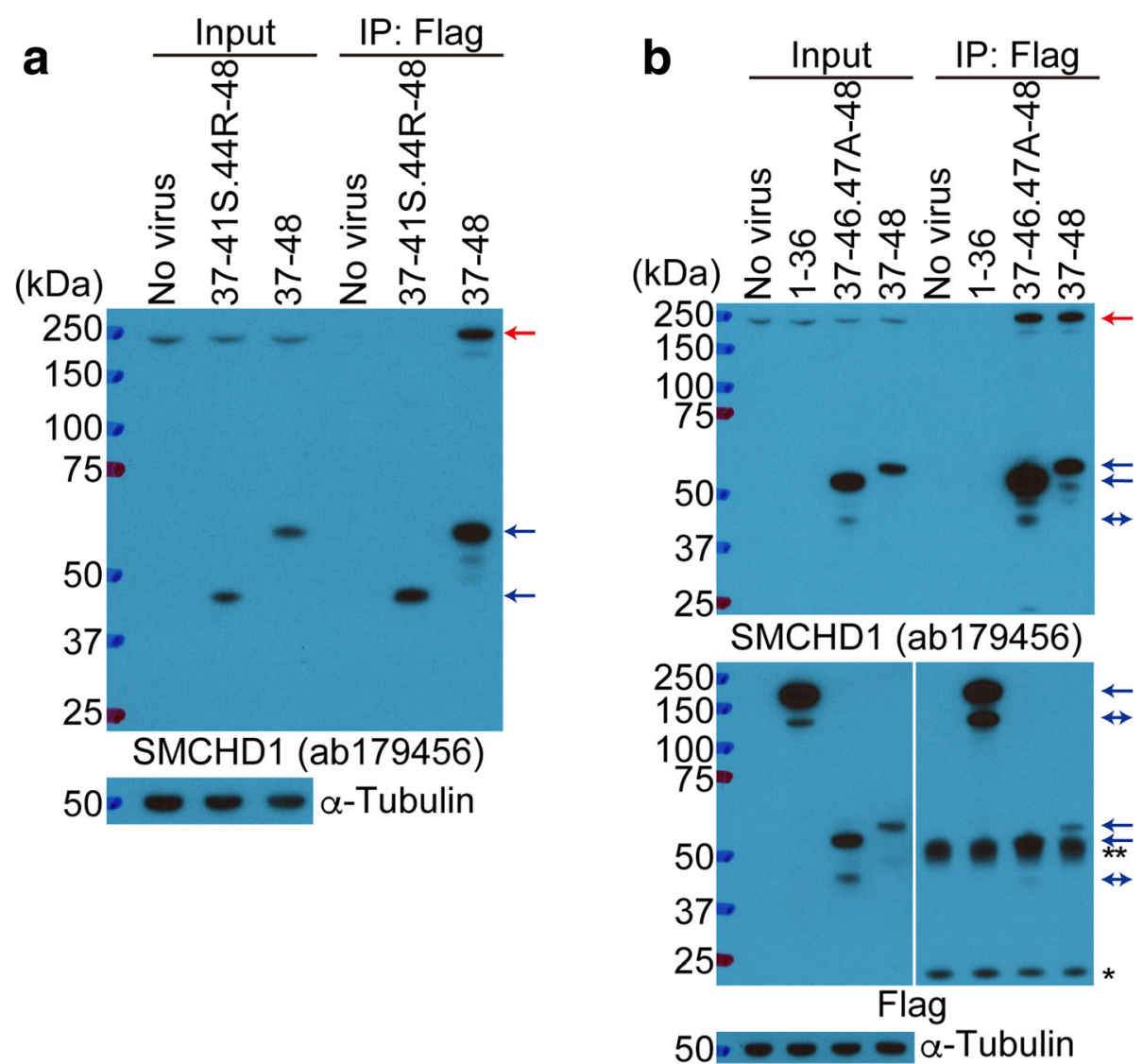

Fig. 4 Identification of regions necessary for SMCHD1 homo-dimerization. a, b IP of exogenous SMCHD1 in control myoblasts transduced with Flag-tagged SMCHD1 lentivirus followed by Western blot with an anti-SMCHD1 antibody (ab179456) detecting the carboxy-terminal region of SMCHD1. For confirming the expression of Exon1-36-Flag, anti-Flag antibody was used after stripping. a-Tubulin was used as a loading control. Red and blue arrows identify endogenous and exogenous SMCHD1, respectively. Blue double arrows identify smaller fragments of exogenous SMCHD1. IgG heavy chain $\left(^{*}\right)$ and light chain $\left(^{*}\right)$ IP (immunoprecipitation)

expressed mostly in the nucleus (Fig. 2c). Similarly, Exon37-48-Flag was expressed mostly in the nucleus, while Exon37-46.47A-48-Flag, which lacks the NLS, was expressed in the cytoplasm (Fig. 2c). Moreover, we deleted this NLS from full-length SMCHD1 (Exon1-46.47A-48-Flag) and confirmed that it was expressed in the cytoplasm (Fig. 2d), which suggests that this NLS is necessary for SMCHD1 to localize to the nucleus. As these 32 amino acids include the consensus sequence of $K(K / R) X(K / R)$ for a classical NLS (bold in the sequence above) [18], we deleted these four amino acids (KRMR) from full-length SMCHD1 ( $\triangle K R M R)$ and confirmed that it was expressed in the cytoplasm (Fig. 2d). A FSHD2 causing mutation of SMCHD1 introduces a stop codon between the hinge domain and the nuclear localization signal (R1868*) [19] and expression of a tagged version of this mutation (R1868*-Flag) showed mostly cytoplasmic localization (Fig. 2d).

In control (MB135) myotubes in differentiation medium, SMCHD1 was mostly localized to one single spot in the nucleus (Fig. 3a). Taking into account that MB135 muscle cells were derived from a female, we tested whether the single spot in myotube nuclei could be co-localized with the inactive $\mathrm{X}$ chromosome marker H3K27me3 (histone $\mathrm{H} 3$ trimethyl lysine 27) $[20,21]$ and determined that the spots for SMCHD1 and H3K27me3 were co-localized (Fig. 3b). Similarly, the expression of Exon1-48-Flag also localized to nuclear foci, although the overexpression showed some additional homogeneous nuclear staining. In contrast, all of the mutants Exon1-36.47M-48-Flag, Exon3748-Flag, and Exon1-9.41-48-Flag showed only diffuse nuclear staining with no evident foci (Fig. 3c), suggesting that the structural integrity of SMCHD1, rather than a single specific region, was necessary for the foci and presumably binding to the inactive $\mathrm{X}$ chromosome.

\section{Identification of regions necessary for SMCHD1 homo- dimerization}

Previous studies using IP showed that the hinge domain was important for SMCHD1 homo-dimerization $[8,10]$. We used IP to determine whether different SMCHD1 mutants would dimerize with endogenous SMCHD1. 

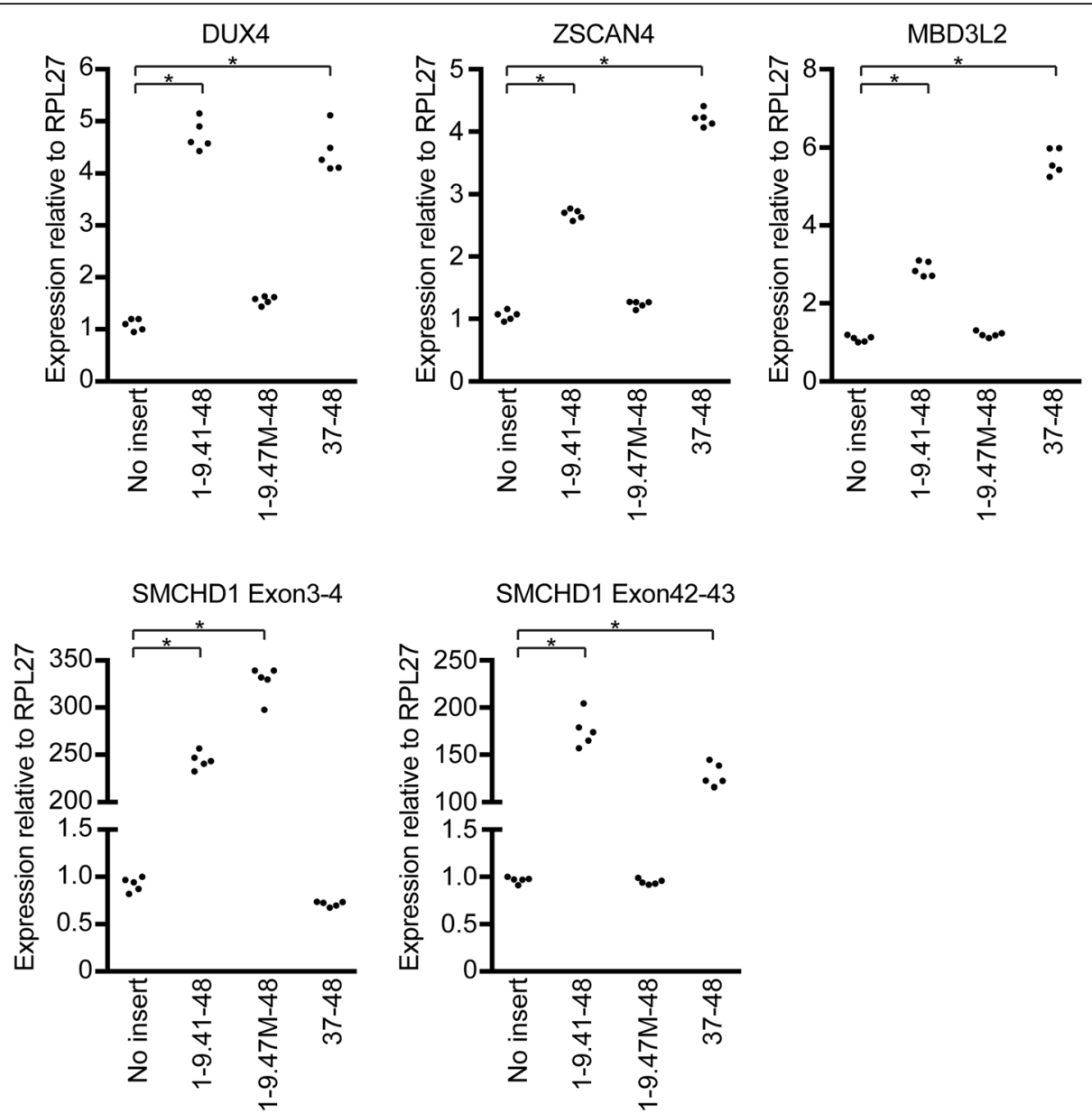

Fig. 5 DUX4 expression in FSHD1 myoblasts with mutant SMCHD1. RT-qPCR for DUX4, ZSCAN4, MBD3L2, and SMCHD1 in FSHD1 myoblasts transduced with different mutant SMCHD1 constructs. RPL27 was used as an internal control. ( $n=5$ in each group) Dunnett' $s$ test $\left({ }^{*} P<0.01\right)$. These are representative data from three experiments

Confirming the prior results, Exon37-48-Flag bound to endogenous SMCHD1, whereas Exon37-41S.44R-48-Flag, which lacks the hinge domain, did not bind to endogenous SMCHD1 (Fig. 4a). Moreover, Exon1-36-Flag, which does not have a hinge domain, also did not bind to endogenous SMCHD1, whereas Exon37-46.47A-48-Flag, which lacks the NLS but has the hinge domain, also bound to endogenous SMCHD1 (Fig. 4b). These results indicate that the hinge domain can mediate dimerization between endogenous SMCHD1 and the mutant SMCHD1 proteins. It is interesting to note that the expression of these mutant SMCHD1 proteins did not alter the abundance of the endogenous SMCHD1 protein, whether the mutant contained the hinge domain or the NLS, or not (Fig. 4a, b), indicating that mutations in SMCHD1 might not alter the abundance of the endogenous SMCHD1 in FSHD2 but might alter its function by forming inactive heterodimers.

To determine whether a mutant SMCHD1 capable of forming a heterodimer with the wild-type might partly interfere with normal function, we overexpressed
SMCHD1 mutants in FSHD1 myoblasts and measured the level of DUX4 expression. Exon1-9.41-48-Flag and Exon37-48-Flag increased DUX4 and DUX4 target genes (ZSCAN4 and MBD3L2) expression, whereas Exon1-9.47M-48-Flag had less effect on DUX4 and its target genes expression (Fig. 5).

\section{Identification of cleavage sites in the SMCHD1 protein}

Unexpectedly, in addition to the band of predicted molecular size for the mutant SMCHD1 proteins, we also detected a smaller band(s) (see double arrows in Fig. 4b). To better understand how the smaller bands were produced, we focused on the small band derived from Exon1-36-Flag. We first determined whether the smaller fragment might represent a cleavage product of SMCHD1 comparing immunoreactivity to antibodies directed to either the amino- or carboxy-terminus of the protein. Antibody HPA039441 detected both the predicted full-length protein $(173.9 \mathrm{kDa})$ and a prominent smaller band (about $50 \mathrm{kDa}$ ), whereas the anti-Flag antibody detected the predicted full-length protein 

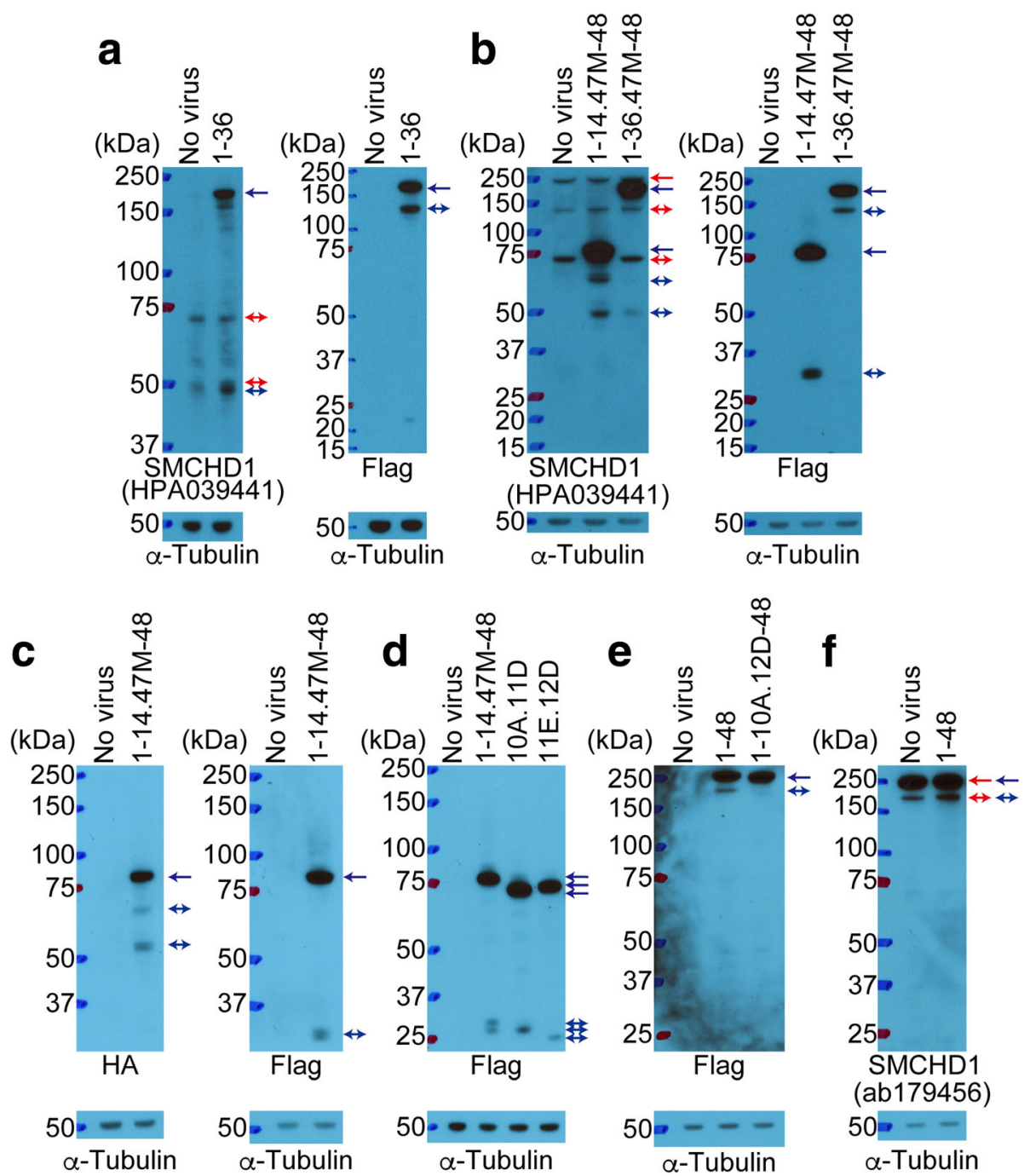

Fig. 6 Identification of the cleavage sites. $\mathbf{a}, \mathbf{b}$, and $\mathbf{d}-\mathbf{f}$ Western blot for SMCHD1 (HPA039441 and ab179456) and Flag in control myoblasts transduced with Flag-tagged SMCHD1. (c) Western blot for HA and Flag in control myoblasts transduced with HA-Exon1-14.47M-48-Flag. aTubulin was used as a loading control. Red and blue arrows indicate endogenous and exogenous SMCHD1, respectively. Red and blue double arrows indicate smaller fragments of endogenous and exogenous SMCHD1, respectively. 10A.11D (Exon1-10A.11D-14.47M-48). 11E.12D (Exon111E.12D-14.47M-48). The smaller band from full length was confirmed by repeat experiments

and a smaller band (about $125 \mathrm{kDa}$ ) (Fig. 6a). The total size of the two smaller bands, 50 and $125 \mathrm{kDa}$, would add together to become a predicted size of the full-length protein. Similar to this, we confirmed the total size of the smaller bands adds to the size of the full-length band in Exon1-36.47M-48-Flag (Fig. 6b).

To narrow down the location of a potential cleavage site, we tested Exon1-14.47M-48-Flag. The HPA039441 detected the predicted band $(82.4 \mathrm{kDa})$ and two smaller bands (approximately 50 and $60 \mathrm{kDa}$ ), whereas the anti-Flag antibody detected the full-length band and a smaller band (about $30 \mathrm{kDa}$ ) (Fig. 6b). Similarly, the added sizes of the smaller bands ( 50 and $30 \mathrm{kDa}$ ) match the size of the full-length band. Moreover, we tested
HA-Exon1-14.47M-48-Flag, which has the HA tag in the amino-terminus and the Flag tag in the carboxy-terminus. Similar to Exon1-14.47M-48-Flag, we detected smaller bands using the anti-HA or Flag antibody (Fig. 6c).

To further narrow down the site(s) of cleavage, we tested Exon1-10A.11D-14.47M-48-Flag (10A.11D), which lacks the 44 aa between 409 and 452 aa in the area of cleavage, and Exon1-11E.12D-14.47M-48-Flag (11E.12D), which lacks the 37 aa between 453 and 489 aa. The anti-Flag antibody detected two smaller bands in Exon1-14.47M-48-Flag, whereas it detected only a single smaller band (about $30 \mathrm{kDa}$ ) in 10A.11D and 11E.12D (Fig. 6d). These results indicate that there might be one cleavage site between 409 and 452 aa and a second site 
between 453 and 489 aa. Next, we tested Exon1-10A.12D-48-Flag, which lack these cleavage sites from full length, and investigated whether there is any difference between Exon1-48-Flag and Exon1-10A.12D-48-Flag. We detected a smaller band from Exon1-48-Flag not Exon1-10A.12D-48-Flag (Fig. 6e). Moreover, antibody ab179456, which recognizes the carboxy-terminus, detected a smaller band in not only Exon1-48-Flag but also endogenous SMCHD1 (Fig. 6f). Together, these results suggest that specific cleavage sites in the ectopic constructs could be also used in the endogenous SMCHD1.

\section{Discussion}

The ATPase domain and the hinge domain make up only $15 \%$ of the total protein, and it is important to progressively annotate additional functional domains to help identify functionally significant polymorphisms. In this study, we identified the nuclear localization signal, confirmed the dimerization domain, and identified cleavage sites in the SMCHD1 protein. The NLS of SMCHD1 was mapped from aa 1961 to 1992 . The four residues (KRMR) in the 32 amino acids is consistent with the consensus sequence of $K(K / R) X(K / R)$ for a classical NLS [18]. In addition to nuclear localization, the localization of SMCHD1 was observed as a bright intra-nuclear spot that co-localized with a marker for the inactive X chromosome [10, 13-15].

Consistent with previous studies $[8,10]$, the hinge domain was important for dimerization between endogenous and exogenous SMCHD1. Since a variety of mutations in SMCHD1 coding region have been reported that have the potential to produce different portions of the SMCHD1 protein and might act as either haploinsufficient or possibly dominant negatives $[4,19$, 22], understanding the domains that confer functional interactions, such as NLS and homo-dimerization, will be important to elucidate the molecular mechanisms for the mutant SMCHD1 regulation of DUX4 expression. In this regard, it is interesting to note that based on our results with different SMCHD1 proteins, the amount of endogenous SMCHD1 was not altered regardless of the presence or absence of a hinge domain in the mutant; however, it is possible that the dimerization with a mutant partner might alter the function of the wild-type SMCHD1 because overexpression of mutants containing the hinge domain resulted in increased expression of DUX4.

A previous study showed that a mutation affecting the activity of the ATPase domain (E147A) or a deletion of the hinge domain failed to localize to the inactive $\mathrm{X}$ chromosome in Smchd1 $1^{-/-}$female mouse embryonic fibroblasts [10]. Together with our finding that deletion of multiple different domains also results in a protein that does not localize to a nuclear focus suggests that lenti-viral expression of SMCHD1 might be an approach to probe the functional significance of polymorphisms of unknown significance in SMCHD1.

As an additional finding, we identified cleavage sites in the SMCHD1 protein between aa 409-452 and aa 453489. We were unable to determine the protease that cleaves at this site. In addition, since we detected additional smaller fragments from the endogenous SMCHD1 (see Fig. 6a, b), it is possible that SMCHD1 might have additional cleavage sites. Further investigation is necessary to determine whether these cleavage sites could be involved in the biological regulation of SMCHD1 degradation.

\section{Conclusions}

We identified regions of SMCHD1 necessary for nuclear localization, confirmed the region necessary for dimerization, and identified cleavage sites using lenti-viral vectors that express Flag-tagged full-length or different mutant SMCHD1 proteins.

\section{Additional files}

Additional file 1: Sequences of the primers for constructions. (XLSX $23 \mathrm{~kb}$ ) Additional file 2: Sequences of the primers for RT-qPCR. (XLSX 46 kb)

\section{Abbreviations}

10A.11D: Exon1-10A.11D-14.47M-48-Flag; 11E.12D: Exon1-11E.12D-14.47M-48Flag; aa: Amino acid; FSHD: Facioscapulohumeral muscular dystrophy; IP: Immunoprecipitation; NLS: Nuclear localization signal

\section{Acknowledgements}

pLenti CMV GFP Puro (658-5) was a gift from Eric Campeau and Paul Kaufman. pMD2.G and psPAX2 were gifts from Didier Trono.

\section{Funding}

This work was supported by the FSH Society and FSHD Canada Foundation $(\mathrm{YH})$.

\section{Availability of data and materials}

All data generated or analyzed during this study are included in this published article and additional files.

\section{Authors' contributions}

$\mathrm{YH}$ and SJT designed the experiments and wrote the manuscript. $\mathrm{YH}$ performed the experiments. Both authors read and approved the final manuscript.

\section{Ethics approval and consent to participate}

This study used pre-existing de-identified human cell lines and was determined not to be Human Subjects Research by the Fred Hutchinson Cancer Research Center Institutional Review Board.

\section{Consent for publication}

Not applicable.

\section{Competing interests}

The authors declare that they have no competing interests.

\section{Publisher's Note}

Springer Nature remains neutral with regard to jurisdictional claims in published maps and institutional affiliations. 
Received: 29 March 2018 Accepted: 24 July 2018

Published online: 02 August 2018

\section{References}

1. Daxinger L, Tapscott SJ, van der Maarel SM. Genetic and epigenetic contributors to FSHD. Curr Opin Genet Dev. 2015;33:56-61.

2. Wijmenga C, Hewitt JE, Sandkuijl LA, Clark LN, Wright TJ, Dauwerse $H G$, et al. Chromosome $4 q$ DNA rearrangements associated with facioscapulohumeral muscular dystrophy. Nat Genet. 1992;2:26-30.

3. Lemmers RJLF, Van Der Vliet PJ, Klooster R, Sacconi S, Camaño P, Dauwerse $J G$, et al. A unifying genetic model for facioscapulohumeral muscular dystrophy. Science. 2010;329:1650-3.

4. Lemmers RJLF, Tawil R, Petek LM, Balog J, Block GJ, Santen GWE, et al. Digenic inheritance of an SMCHD1 mutation and an FSHD-permissive D4Z4 allele causes facioscapulohumeral muscular dystrophy type 2. Nat Genet 2012:44:1370-4

5. Sacconi S, Lemmers RJLF, Balog J, Van Der Vliet PJ, Lahaut P, Van Nieuwenhuizen MP, et al. The FSHD2 gene SMCHD1 is a modifier of disease severity in families affected by FSHD1. Am J Hum Genet. 2013;93:744-51.

6. Jansz N, Chen K, Murphy JM, Blewitt ME. The epigenetic regulator SMCHD1 in development and disease. Trends Genet. 2017;33:233-43.

7. Chen K, Hu J, Moore DL, Liu R, Kessans SA, Breslin K, et al. Genome-wide binding and mechanistic analyses of Smchd1-mediated epigenetic regulation. Proc Natl Acad Sci U S A. 2015;112:E3535-44.

8. Chen K, Czabotar PE, Blewitt ME, Murphy JM. The hinge domain of the epigenetic repressor Smchd1 adopts an unconventional homodimeric configuration. Biochem J. 2016;473:733-42.

9. Chen K, Dobson RCJ, Lucet IS, Young SN, Pearce FG, Blewitt ME, et al. The epigenetic regulator Smchd1 contains a functional GHKL-type ATPase domain. Biochem J. 2016;473:1733-44.

10. Brideau NJ, Coker H, Gendrel A, Siebert CA, Bezstarosti K, Demmers J, et al. Independent mechanisms target SMCHD1 to trimethylated histone $\mathrm{H} 3$ lysine 9-modified chromatin and the inactive X chromosome. Mol Cell Biol. 2015;35:4053-68.

11. Gordon CT, Xue S, Yigit G, Filali H, Chen K, Rosin N, et al. De novo mutations in SMCHD1 cause Bosma arhinia microphthalmia syndrome and abrogate nasal development. Nat Genet. 2017;49:249-55.

12. Blewitt ME, Vickaryous NK, Hemley SJ, Ashe A, Bruxner TJ, Preis Jl, et al. An $\mathrm{N}$-ethyl-N-nitrosourea screen for genes involved in variegation in the mouse. Proc Natl Acad Sci U S A. 2005;102:7629-34.

13. Blewitt ME, Gendrel A-V, Pang Z, Sparrow DB, Whitelaw N, Craig JM, et al. SmcHD1, containing a structural-maintenance-of-chromosomes hinge domain, has a critical role in X inactivation. Nat Genet. 2008;40:663-9.

14. Gendrel A, Apedaile A, Coker H, Termanis A, Zvetkova I, Godwin J, et al. Smchd1-dependent and -independent pathways determine developmental dynamics of $\mathrm{CpG}$ island methylation on the inactive $\mathrm{X}$ chromosome. Dev Cell. 2012;23:265-79.

15. Nozawa R-S, Nagao K, Igami K-T, Shibata S, Shirai N, Nozaki N, et al. Human inactive $X$ chromosome is compacted through a PRC2independent SMCHD1-HBiX1 pathway. Nat Struct Mol Biol. 2013;20: 566-73.

16. Mason AG, Slieker RC, Balog J, Lemmers RJLF, Wong CJ, Yao Z, et al. $\mathrm{SMCHD1}$ regulates a limited set of gene clusters on autosomal chromosomes. Skelet Muscle. 2017;7:12.

17. Campeau E, Ruhl VE, Rodier F, Smith CL, Rahmberg BL, Fuss JO, et al. A versatile viral system for expression and depletion of proteins in mammalian cells. PLoS One. 2009;4:e6529.

18. Lange A, Mills RE, Lange CJ, Stewart M, Devine SE, Corbett AH. Classical nuclear localization signals: definition, function, and interaction with importin alpha. J Biol Chem. 2007;282:5101-5.

19. Lemmers RJLF, Goeman JJ, Van der Vliet PJ, Van Nieuwenhuizen MP, Balog J, Vos-Versteeg M, et al. Inter-individual differences in CpG methylation at D4Z4 correlate with clinical variability in FSHD1 and FSHD2. Hum Mol Genet. 2015;24:659-69.

20. Plath K, Fang J, Mlynarczyk-Evans SK, Cao R, Worringer KA, Wang H, et al. Role of histone H3 lysine 27 methylation in X inactivation. Science. 2003; 300:131-5.
21. Silva J, Mak W, Zvetkova I, Appanah R, Nesterova TB, Webster Z, et al. Establishment of histone $\mathrm{H} 3$ methylation on the inactive $X$ chromosome requires transient recruitment of Eed-Enx1 polycomb group complexes. Dev Cell. 2003:4:481-95.

22. Larsen M, Rost S, El Hajj N, Ferbert A, Deschauer M, Walter MC, et al. Diagnostic approach for FSHD revisited: SMCHD1 mutations cause FSHD2 and act as modifiers of disease severity in FSHD1. Eur J Hum Genet. 2015; 23:808-16.

Ready to submit your research? Choose BMC and benefit from

- fast, convenient online submission

- thorough peer review by experienced researchers in your field

- rapid publication on acceptance

- support for research data, including large and complex data types

- gold Open Access which fosters wider collaboration and increased citations

- maximum visibility for your research: over $100 \mathrm{M}$ website views per year

At BMC, research is always in progress.

Learn more biomedcentral.com/submissions 